\title{
Comparison of the efficacy of a demand oxygen delivery system with continuous low flow oxygen in subjects with stable COPD and severe oxygen desaturation on walking
}

Department of Thoracic Medicine, London Chest Hospital, Bonner Road, London E2 9JX, UK C M Roberts J Bell

J A Wedzicha

Correspondence to: Dr C M Roberts, Chest Clinic, Whipps Cross Hospital, London E11 1NR UK.

Received 21 August 1995 Returned to authors 14 November 1995 Revised version received 14 December 1995 Accepted for publication 12 March 1996
Abstract

Background - Provision of ambulatory oxygen using an intermittent pulsed flow regulated by a demand oxygen delivery system (DODS) greatly increases the limited supply time of standard portable gaseous cylinders. The efficacy of such a system has not previously been studied during submaximal exercise in subjects with severe chronic obstructive pulmonary disease (COPD) in whom desaturation is likely to be great and where usage is often most appropriate.

Methods - Fifteen subjects with severe COPD and oxygen desaturation underwent six minute walk tests performed in random order to compare the efficacy of a demand oxygen delivery system (DODS) with continuous flow oxygen. Walk distance, breathlessness, oxygen saturation, resting time, and recovery time (objective and subjective) were recorded and compared for each walk.

Results - Breathing continuous oxygen compared with baseline air breathing improved mean walk distance ( $295 \mathrm{~m}$ versus $271 \mathrm{~m}$ ) and recovery time (47 seconds versus 112 seconds), whilst the lowest recorded saturation $(81 \%$ versus $74 \%$ ) and time desaturated below $90 \%$ (201 seconds versus 299 seconds) were reduced. When the DODS was compared with air breathing only the walk distance changed $(283 \mathrm{~m}$ versus $271 \mathrm{~m}$ ). A comparison of the DODS with continuous oxygen breathing showed the DODS to be less effective at oxygenating subjects with inferior lowest saturation $(78 \%$ versus $81 \%)$, time spent below $90 \%$ (284 seconds versus 201 seconds), time to objective recovery ( 83 seconds versus 47 seconds), and walk distance ( $283 \mathrm{~m}$ versus $295 \mathrm{~m}$ ).

Conclusions - Neither of the delivery systems was able to prevent desaturation in these subjects. The use of continuous flow oxygen, however, was accompanied by improvements in oxygenation, walk distance, and recovery time compared with air breathing. The DODS produced only a small increase in walk distance without elevation of oxygen saturation, but was inferior to continuous flow oxygen in most of the measured variables when compared directly.

(Thorax 1996;51:831-834)
Keywords: demand oxygen delivery system, ambulatory oxygen, chronic obstructive pulmonary disease.

Ambulatory oxygen may be used in subjects with stable chronic obstructive pulmonary disease (COPD) to relieve symptoms of breathlessness on exertion, improve mobility, or to extend the use of long term oxygen therapy. ${ }^{12}$ Despite these broad indications, relatively few patients in the UK with COPD receive this form of treatment. One important reason for this is the short duration of supply provided by the ambulatory cylinder. ${ }^{2}{ }^{3}$ The standard UK system is a gaseous cylinder of 220-240 litres capacity which, if used at the conventional flow rate of $21 / \mathrm{min}$, will provide oxygen for a maximum of two hours and often less because of incomplete cylinder filling.

One alternative is to use an intermittent flow demand oxygen delivery system (DODS). ${ }^{4}$ Such a device interrupts the supply from the cylinder source and releases a bolus of oxygen at the beginning of inspiration. Demand oxygen delivery devices have been shown to extend the supply of gaseous cylinder and liquid oxygen by up to seven times that of the continuous flow system. ${ }^{5}$ The ability of the DODS to extend the oxygen supply beyond that of a continuous flow cylinder is not in doubt. The comparable oxygenation provided by the two systems in subjects with COPD who severely desaturate during submaximal exercise has not, however, previously been investigated. This study therefore aimed to compare oxygenation, walk distance, and perceived breathlessness using these two ambulatory oxygen systems.

\section{Methods}

All outpatients with COPD attending the Department of Thoracic Medicine at the London Chest Hospital during a nine month period who were referred for an ambulatory oxygen assessment or who were already receiving this treatment were considered for the study. Selection and prescription of ambulatory oxygen therapy was according to previously reported guidelines. ${ }^{7}$ Those subjects whose oxygen saturation $\left(\mathrm{SaO}_{2}\right)$ fell significantly (below 90\%) during a screening six minute walk test while breathing air were included in the study.

Those selected were judged clinically to be in a stable state. Each subject underwent spiro- 
metric testing and resting arterial blood gas analysis using earlobe arterialised capillary sampling. ${ }^{89}$ Once entered into the study subjects underwent a further practice six minute walk test and were then randomised, using a Latin squares sequence, to undergo four further walks with a 40 minute rest period between each. The four walks were: (1) baseline, (2) breathing air and carrying a portable cylinder and DODS device, (3) breathing oxygen from the DODS set at the equivalent of $2 \mathrm{l} / \mathrm{min}$ by the manufacturer's standards, and (4) breathing continuous flow oxygen at a rate of $2 \mathrm{l} / \mathrm{min}$. Each walk was supervised by the same operator (JB).

Continuous oxygen was delivered by nasal cannulae at a rate of $21 / \mathrm{min}$ from a portable 230 litre cylinder (Sabre, Aldershot, UK) which has a duration of supply of about 115 minutes and weighs $2.9 \mathrm{~kg}$. Demand oxygen was delivered by one of the most efficient of the DODS, ${ }^{10}$ the Chad Oxymatic Model 201-A (Chad Therapeutics Inc., Chatsworth, USA), now available in the UK through a local distributor (Life Support Europe, Dunstable, UK). The oxygen for this system was supplied by nasal cannulae from a 185 litre cylinder (Chad Inc., Chatsworth, USA), weighing 1.25 $\mathrm{kg}$, at an equivalent flow of $21 /$ min determined by the Oxymatic DODS (weight $0.28 \mathrm{~kg}$ ) which gives a duration of supply of about 10.5 hours.

The Chad Oxymatic (fig 1) is a small battery powered electronic device which delivers a metered volume $35 \mathrm{ml}$ pulse of oxygen in 200 ms immediately on sensing the beginning of inspiration. The device uses standard nasal cannulae fitted to a sensing circuit which controls a solenoid valve. A setting at which the valve is opened on every second breath sensed provides an oxygen supply equivalent to that of $21 /$ min continuous oxygen. This setting can be altered from sensing every fourth to every single breath, providing an equivalent range of 1-4 $1 / \mathrm{min}$ continuous flow (manufacturer's recommendation).

Before each test the subjects completed a visual analogue scale of breathlessness (VAS) consisting of a $100 \mathrm{~mm}$ horizontal line ${ }^{11}$ labelled "not at all breathless" at one end and "the most breathless I have ever been" at the other. They were instructed to mark on the line the point which best described their state of breathlessness at that time. This test was repeated immediately upon completion of each walk. A value "change in VAS after exercise" was calculated as the difference between the two points. In addition, their respiratory and heart rates were recorded before and upon completion of each test walk. The difference in respiratory rate before and after the walk was calculated and termed "change in respiratory rate". Pulse oximetry using the Minolta Pulseox-7 (AVL Medical Instruments Ltd, Schaffhausen, Switzerland) was recorded throughout each walk with the baseline before administration of oxygen, lowest saturation, and time desaturated below $90 \%$ being noted. Subjective recovery time was judged by the subjects themselves and an "objective" recovery time was recorded by the observer based upon a return of oxygen saturation, heart and respiratory rates to baseline levels. The number of stops, time spent resting, and total distance walked were recorded.

The protocol was approved by the ethics committee of the Royal Brompton National Heart and Lung Hospitals.

\section{STATISTICAL ANALYSIS}

Analysis was performed using the SPSS/PC + programme (SPSS Inc., Chicago, USA). Descriptive data were first produced. Analysis of variance was used to ensure equivalent baseline data. Paired data between different walks was analysed using the Student's paired $t$ test or the Wilcoxon rank sum test for non-parametric data.

\section{Results}

Sixteen patients were referred or attended the outpatient department for ambulatory oxygen therapy during this period and desaturated below $90 \%$ on their screening walk. One of these subjects failed to desaturate to this extent on any of the subsequent walks and was therefore not considered in the analysis of data. The 15 remaining subjects (nine men), of mean (SD) age 67.5 (6.2) years, range 58-79, had mean (SD) spirometric and arterial blood gas data as follows: forced expiratory volume in one second $\left(\mathrm{FEV}_{1}\right) 0.85$ (0.29) l (31 (8.1)\% predicted), forced vital capacity (FVC) 2.14 (0.63) 1 (62 (13.5)\% predicted), $\mathrm{PaO}_{2} 6.98$ (0.96) kPa, $\mathrm{PaCO}_{2} 6.65$ (1.09) kPa

There was no statistical difference in the baseline values of respiratory rate, VAS, or oxygen saturation breathing air before any of the four walk tests. Paired data analysis demon-

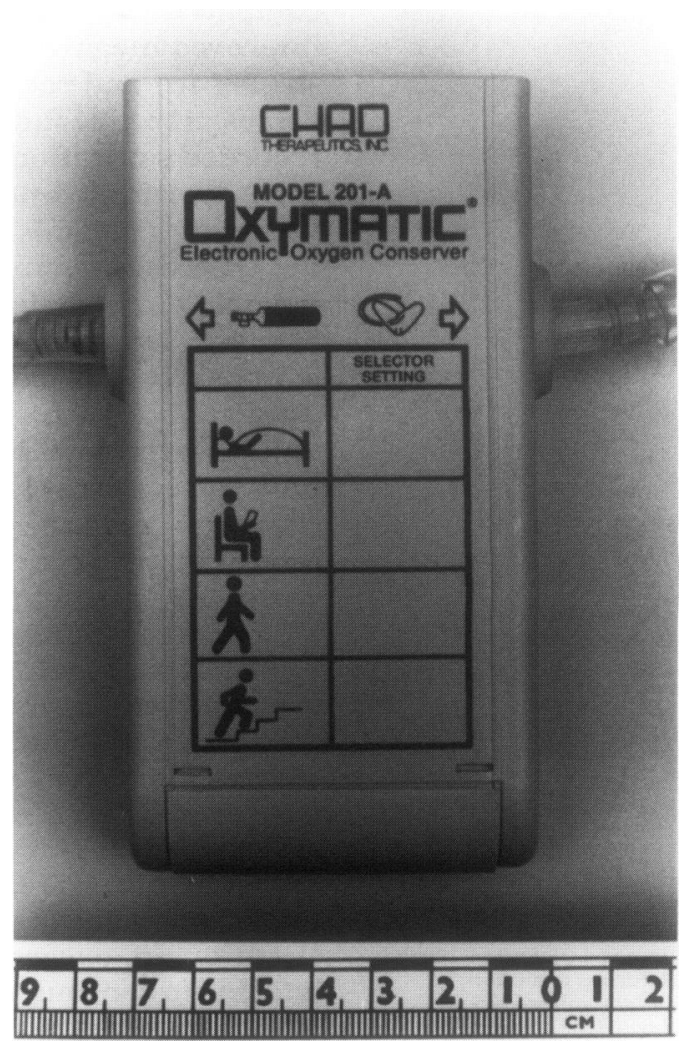

Figure 1 The Chad Oxymatic demand oxygen delivery device. 
Table 1 Comparison of mean (SD) values for the baseline walk (1), walk carrying the DODS and cylinder disconnected (2), walk using the DODS (3), and walk using continuous flow oxygen (4)

\begin{tabular}{|c|c|c|c|c|c|c|c|c|c|}
\hline Value & $\begin{array}{l}\text { Lowest } \\
\text { saturation (\%) }\end{array}$ & $\begin{array}{l}\text { Time }<90 \% \\
\text { saturated }(s)\end{array}$ & $\begin{array}{l}\text { Walk distance } \\
\text { (m) }\end{array}$ & $\begin{array}{l}\text { Change in } \\
\operatorname{VAS}(\mathrm{mm})\end{array}$ & $\begin{array}{l}\text { Time to objective } \\
\text { recovery }(s)\end{array}$ & $\begin{array}{l}\text { Time to } \\
\text { subjective } \\
\text { recovery (s) }\end{array}$ & $\begin{array}{l}\text { Change in } \\
\text { respiratory } \\
\text { rate (bpm) }\end{array}$ & $\begin{array}{l}\text { Time } \\
\text { resting (s) }\end{array}$ & No. of rests \\
\hline Baseline (1) & $74.6(11.3)$ & $299(78)$ & $271(91.5)$ & $44.7(23.8)$ & $112(62)$ & $138(52)$ & $7.1(5.3)$ & $32.8(49.6)$ & $0.87(1.1)$ \\
\hline $\begin{array}{l}\text { Carrying DODS } \\
\text { and cylinder (2) }\end{array}$ & $73.9(12.1)$ & $290(77.9)$ & $275.1(95.7)$ & $46.5(33.7)$ & $128.3(49.8)$ & $156.3(64.1)$ & $7.4(5.2)$ & $42.9(66.5)$ & $0.8(1.0)$ \\
\hline DODS (3) & $78.0(9.8)$ & $284(81)$ & $283.3(88.8)$ & $37.3(34.2)$ & $82.7(54)$ & $149(85)$ & $5.8(4.5)$ & $38.6(66.1)$ & $0.73(0.96)$ \\
\hline Continuous $\mathrm{O}_{2}(4)$ & $81(9.6)$ & $201(39)$ & $295.4(89.1)$ & $35.3(34.5)$ & $47(47)$ & $111(47)$ & $6.1(4.1)$ & $24.9(47.9)$ & $0.47(0.83)$ \\
\hline $\mathrm{p}$ value $(\mathrm{CI}) 1$ vs 4 & $\begin{array}{l}0.004 \\
(2.42 \text { to } 10.4)\end{array}$ & $\begin{array}{l}0.008 \\
(30.2 \text { to } 167.0)\end{array}$ & $\begin{array}{l}0.003 \\
(9.5 \text { to } 39.3)\end{array}$ & NS & $\begin{array}{l}0.001 \\
(33.4 \text { to } 99.6)\end{array}$ & $\begin{array}{l}0.03 \\
(3.21 \text { to } 53.2)\end{array}$ & NS & NS & NS \\
\hline $\mathrm{p}$ value $(\mathrm{CI}) 2$ vs 4 & $\begin{array}{l}0.002 \\
(1.24 \text { to } 4.36)\end{array}$ & $\begin{array}{l}0.003 \\
(32.7 \text { to } 135.0)\end{array}$ & $\begin{array}{l}0.048 \\
(0.1 \text { to } 24.0)\end{array}$ & NS & $\begin{array}{l}0.001 \\
(-7.47 \text { to } 66.8)\end{array}$ & NS & NS & NS & NS \\
\hline
\end{tabular}

VAS = visual analogue scale; DODS = demand oxygen delivery system.

strated no statistical difference in any of the values measured between the baseline walk while breathing air and the walk carrying the DODS device and portable cylinder whilst breathing air.

A comparison of values measured while breathing $2 \mathrm{l} / \mathrm{min}$ continuous oxygen with the baseline walk while breathing air showed statistically significant differences in walk distance, subjective time to recovery, objective time to recovery, lowest recorded $\mathrm{SaO}_{2}$, and time spent under $90 \% \mathrm{SaO}_{2}$ (table 1). A smaller change in VAS following exercise did not reach statistical significance, and time spent resting, number of rests, and change in respiratory rate were also similar with and without oxygen.

When these same measurements were compared for the Oxymatic device set at the equivalent of $2 \mathrm{l} / \mathrm{min}$ oxygen with baseline, walk distance was significantly improved using the DODS (283.3 $\mathrm{m}$ versus $271.0 \mathrm{~m}$ (CI 1.6 to $23.1), p<0.05)$ and change in respiratory rate after exercise showed a small difference (mean 6 versus 6.6 (CI -2.5 to 0$), \mathrm{p}<0.05$, Wilcoxon rank sum test). No other measured variable was significantly different on these two test walks.

Comparison of the DODS device set at 2 $1 / \mathrm{min}$ with continuous flow oxygen set at 2 $1 /$ min showed that the lowest recorded saturation and time spent desaturated were both statistically inferior for the Oxymatic (table 1).

\section{Discussion}

This study has shown that the Oxymatic demand oxygen delivery device failed to achieve oxygenation equivalent to that of continuous oxygen therapy during submaximal exercise in subjects with COPD and severe arterial oxygen desaturation.

Subjects using the Oxymatic achieved oxygen saturations only marginally higher than when breathing air alone, and these changes failed to reach statistical significance. A comparison of baseline (air breathing) tests with those breathing intermittent flow oxygen showed that only walk distance was significantly improved (283.3 m versus $271.0 \mathrm{~m}$ ). Direct comparison of continuous oxygen with the intermittent flow system showed the former to be superior in maintaining oxygenation and improving walk distance.

These findings contrast with some previous studies in subjects with COPD. Many of these earlier protocols made comparisons between continuous and intermittent flow in the resting state $\mathrm{s}^{12-14}$ and found oxygenation to be similar. The more relevant effect of exercise and the induced desaturation has been less extensively studied in subjects with COPD and the findings are less consistent. In one of the early trials Gould et $a l^{12}$ observed that not all devices were equally efficacious during exercise. Confirmation followed in a much larger contemporary study $^{10}$ in which six different DODS devices were measured against continuous oxygen using a walk test protocol. Some of the DODS devices tested were unable to maintain oxygenation comparable with continuous flow. The methods of triggering and delivering intermittent pulsed oxygen were suggested as critical in determining the efficacy of the individual system. The Oxymatic was examined in this trial and actually maintained oxygenation well in the subjects included.

If studies during exercise are scrutinised it can be observed that the DODS do not maintain oxygenation in all subjects studied even though the group mean results may be impressive. ${ }^{415}$ Various explanations for this have been provided which include changing the respiratory pattern from nasal to mouth breathing during exercise, reduced tidal flow failing to trigger the flow sensor, rapid respiratory rates exceeding the pulse delay of the flow sensor, and pursed lip breathing leading to a local oral reservoir of oxygen when using continuous flow delivery. ${ }^{4}{ }^{10} 17$

One further explanation of the apparent discrepancy between the individual data is the severity of the desaturation in the subject groups studied. Whilst previous investigators have demonstrated predominantly minor degrees of desaturation, ${ }^{5} 18$ in this study profound desaturation occurred in all subjects and, indeed, was one of the selection criteria for consideration of oxygen therapy. So severe was the desaturation that even continuous oxygen at $2 \mathrm{l} / \mathrm{min}$ failed to prevent a degree of desaturation in some of these subjects and most fell below $90 \%$ during their walk while breathing oxygen. In the previously described studies oxygen prevented all but the most severe desaturation. This reflects the selection criteria for this study, which is more likely to represent current practice in the UK in which ambulatory oxygen is used infrequently and is likely to be reserved for the more severely affected patient. ${ }^{19}$ 
It might be suggested that a higher flow rate of oxygen to correct the hypoxaemia completely would have improved subject performance and the Oxymatic may also have been more effective. It was the intention of this study to examine the DODS device under the circumstances of current practice. Although flow rates of $4 \mathrm{l} / \mathrm{min}$ are sometimes used for refractory hypoxaemia, this level of delivery is not practical for the ambulatory gaseous cylinder which would be exhausted within 30 minutes. In addition, McDonnell et al have previously shown that ambulatory oxygenation provided by a DODS device was less efficient at higher equivalent flow rates than at lower ones. Although the greater supply from a DODS might allow an increased flow rate during exercise, the value of this manoeuvre remains unproven in submaximal exercise. ${ }^{20}$

Overall, the DODS device was not as effective as continuous flow supplemental oxygen in limiting submaximal exercise induced desaturation. Although a DODS may provide a longer oxygen supply and benefit less severely hypoxaemic subjects and those at rest, the Oxymatic is not as effective in subjects who severely desaturate with exercise. It is recommended that individual subjects should be tested to ensure benefit before a decision is made to supply an Oxymatic as an alternative to continuous flow oxygen for ambulatory use.

Our thanks to Dr K D MacRae for advice regarding the statistical analysis and to Life Support (Europe) for the loan of the Oxymatic devices used in the study.

1 Howard P, De Haller R. Domiciliary oxygen - by liquid or concentrator? Eur Respir f 1991;4:1284-7.

2 Vergeret J, Brambilla C, Mounier L. Portable oxygen therapy: use and benefit in hypoxaemic COPD patients on long term oxygen therapy. Eur Respir 7 1989;2:20-5.
3 Lock SH, Blowe G, Prynne M, Wedzicha JA. Comparison of liquid and gaseous oxygen for domiciliary portable use. Thorax 1992;47:98-100.
Thuid

4 McDonnell TJ, Wanger JS, Senn S, Cherniak RM. Efficacy of pulsed oxygen delivery during exercise. Respir Care 1986;31:883-8.

5 Tiep BL, Carter R, Nicotra B, Berry J, Phillips RE, Ostap B. Demand oxygen delivery during exercise. Chest 1987; 91:15-20.

6 Carter R, Tashkin D, Djahed B, Hathaway E, Nicotra MB, Tiep BL. Demand oxygen delivery for patients with restrictive lung disease. Chest 1989;96:1307-11.

7 Lock SH, Paul EA, Rudd RM, Wedzicha JA. Portable oxygen therapy: assessment and usage. Respir Med 1991;85: 407-12.

8 Spiro SG, Dowdeswell IRG. Arterialised ear blood samples for blood gas tensions. $\mathrm{Br} \mathcal{F}$ Dis Chest 1976;70:263-8.

9 Pitkin AD, Roberts CM, Wedzicha JA. Arterialised earlobe blood gas analysis: an underused technique. Thorax 1994; 49:364-6.

10 Braun SR, Spratt G, Scott GC, Ellersieck M. Comparison of six oxygen delivery systems for COPD patients at rest of six oxygen delivery systems for COPD p
and during exercise. Chest 1992;102:694-8.

11 Adams L, Lane R, Shea SA, Cockcroft A, Guz A. Breathlessness during different forms of ventilatory stimulation: a study of mechanisms in normal subjects and respiratory patients. Clin Sci 1985;69:663-72.

12 Gould GA, Hayhurst MD, Scott W, Flenley DC. Clinical assessment of oxygen conserving devices in chronic bronassessment of oxygen conserving devices in chro
chitis and emphysema. Thorax 1985;40:820-4.

13 Tiep BL, Christopher KL, Spofford BT, Goodman JR, Worley PD, Macey SL. Pulsed nasal and transtracheal oxygen delivery. Chest 1990;97:364-8.

14 Kerby GR, O'Donohue WJ, Romberger DJ, Hanson FN, Koenig GA. Clinical efficacy and cost benefit of pulse flow oxygen in hospitalized patients. Chest 1990;97:369-72.

15 Monasterio C, Escarrabill J, Barbe F, Estopa R, Manresa F. Evaluacion de la valvula ahorradora de oxigeno durante el esfuerzo. Med Clin 1992;98:128-30.

16 Segard B, Muir JF, Bedicam JM, Defouilly C, Sautegteau A. The quality of oxygen therapy delivery systems using oxygen-saving valves. A multicentre study. Rev Mal Respir 1992;9:197-204.

17 Senn S, Wanger J, Fernandez E, Cherniak RM. Efficacy of a pulsed oxygen delivery device during exercise in patients with chronic respiratory disease. Chest 1989;96:467-72.

18 Bower JS, Brook CJ, Zimmer K, Davis D. Performance of a demand oxygen saver system during rest, exercise, and sleep in hypoxaemic patients. Chest 1988;94:77-80.

19 Pierson DJ. Home respiratory care in different countries. Eur Respir f 1989;2(Suppl 7):630-6.

20 Leach RM, Davidson AC, Chinn S, Twort CHC, Cameron IR, Bateman NT. Portable liquid oxygen and exercise ability in severe respiratory disability. Thorax 1992;47:781-9. 